\title{
Random Walks and the Eigenvalues of Elliptic Difference Equations
}

\author{
Wolfgang Wasow ${ }^{1}$
}

\begin{abstract}
This paper is concerned with the application of Monte Carlo methods to differential equations of the form, $\nabla^{2} u+g(x, y) u=0$. It is shown that under suitable conditions the Monte Carlo solution converges and gives the solution of the difference analog of the above equation.
\end{abstract}

\section{Introduction}

The aim of this paper is to describe and investigate a random walk procedure that can be used to approximate the solutions of elliptic partial differential equations containing the unknown function itself and not only its derivatives. The method also leads to a scheme for the numerical determination of the lowest eigenvalue of such differential equations.

As a computational technique, our method is somewhat similar to the one used by Donsker and Kac $[3]^{2}$ for the calculation of the lowest eigenvalue of Schroedinger's equation, but the underlying theory is more elementary than the theorem on Wiener integrals of [4] used in [3].

Like all computational methods based on random sampling, those described in the present paper require the use of a high-speed calculating machine. Numerical tests are in progress.

The random walks considered lead to difference equations. By virtue of known results [1], [6], the solutions thus obtained are, for small step length, approximations to the corresponding solutions of the limiting differential equations.

Let the symbol $\Delta$ denote the finite difference analogue of the Laplace operator, i. e.,

$$
\begin{aligned}
\Delta y(x, y)= & \frac{1}{h^{2}}[u(x+h, y)+u(x-h, y) \\
& +u(x, y+h)+u(x, y-h)-4 u(x, y)] .
\end{aligned}
$$

Then we shall be concerned with the difference equation

$$
\Delta u+g(x, y) u=0,
$$

where $g(x, y)$ is to be sufficiently regular to guarantee convergence of the solutions considered to those of the corresponding differential equation.

Everything that follows can be easily extended to more than two dimensions. Extensions to other elliptic differential equations are also possible.

In the sequel, the word "point", without further specification, is meant to refer to the points of a square lattice with mesh length $h$.

1 The preparation of this paper was sponsored (in part) by the Office of Naval

Research.
2 Figures in brackets indicate the literature reference at the end of this paper.

\section{Random Walk Procedure}

Let $B$ be the interior of a finite domain whose boundary points form the set $C$. Consider the problem of finding the solution of eq 1 in $B$ which assumes prescribed values $f(S)$ at the points $S$ of $C$. This problem will be shown to be related to the following random walk procedure.

Let $k(P)$ be a positive function defined in $B$. A particle of mass 1 starts from an inner point $P$. Before moving to one of the four neighboring points $P_{j},(j=1, \ldots, 4)$, its mass is multiplied by the value of $k(P)$ at $P$. Then it moves to a neighboring point, all 4 points having the same probability $1 / 4$ of being chosen. After a certain number of steps the particle arrives for the first time at the boundary, say at the point $S$. Consider now the random variable which is equal to the product of the mass upon arrival at the boundary by the value of $f(P)$ at that point. We claim that, for appropriate choice of $k(P)$, the expected value of this random variable - if it is finite is the solution of eq 1 assuming the values $f(S)$ on $C$.

In the description above, the infinite random walks which never reach $C$ have been ignored. This is permissible, since the total probability associated with such walks is zero, (see [1], p. 44).

Without loss of generality we may restrict the discussion to the special boundary values

$$
f(S)=\delta(R, S) \equiv\left\{\begin{array}{l}
1 \text { for } S=R \\
0 \text { for } S \neq R
\end{array}\right.
$$

where $R$ is some fixed arbitrary point of $C$. For, if we denote the expected value upon arrival corresponding to these special boundary values by $E(P, R)$, then the expected value for any boundary function $f(R)$ is $\sum_{R \in C} E(P, R) f(R)$. If $E(P, R)$ is indeed the solution of eq 1 assuming the values $\delta(R, S)$ on the boundary, then it follows immediately that $\sum_{R \in C} E(P, R) f(R)$ is the solution with boundary values $f(S)$.

Theorem 1: Set

$$
k(P)=\left(1-\frac{h^{2}}{4} g(P)\right)^{-1}
$$


and let $h$ be so small that $k(P)>0$ in $B$, then the function $E(P, R)$ described above satisfies - if it is finitewith respect to $P$ the difference $e q 1$ and the boundary condition 2.

Proof: Let $\left\{L_{n}(P, R)\right\}$ be the set of all possible paths starting at $P$ and reaching $C$ at $R$ on the $n$th step. The probability of the particle moving along any such path is $4^{-n}$. Let $m_{n}(P, R)$ be the mass upon arrival corresponding to a given such path and write

$$
e_{n}(P, R)=\frac{1}{4^{n}} \sum_{\left\{L_{n}(P, R)\right\}} m_{n}(P, R)
$$

the summation extended over all paths of $\left\{L_{n}(P, R)\right\}$. This quantity $e_{n}(P, R)$ is the expected mass upon arrival on $C$, if only arrivals at $R$ and on the $n$th step exactly are counted with their actual mass, and all other random walks ending on $C$ are considered to contribute the mass zero. If we set

$$
E_{n}(P, R)=\sum_{\nu=0}^{n} e_{\nu}(P, R)
$$

then

$$
E(P, R)=\lim _{n \rightarrow \infty} E_{n}(P, R)
$$

provided the limit is finite.

Let $P_{j},(j=1, \cdots, 4)$, be the particular neighboring point of $P$ reached by the particle at the first step, when following the particular path $L_{n}(P, R)$; then

$$
m_{n}(P, R)=k(P) m_{n-1}\left(P_{j}, R\right) \text {, for } n>0,
$$

if $m_{n-1}\left(P_{j}, R\right)$ is the mass upon arrival at $R$ corresponding to that path of the set $\left\{L_{n-1}\left(P_{j}, R\right)\right\}$ which is part of $L_{n}(P, R)$. From eq 4 and eq 7 we have

$e_{n}(P, R)=\frac{1}{4^{n}} \sum_{j=1}^{4} \sum_{\left\{L_{n-1}\left(P_{j}, R\right)\right\}} k(P) m_{n-1}\left(P_{j}, R\right), n>0$,

and, therefore,

$$
e_{n}(P, R)=\frac{k(P)}{4} \sum_{j=1}^{4} e_{n-1}\left(P_{j}, R\right), n>0 .
$$

By means of definition 5 this yields

$$
E_{n}(P, R)=\frac{k(P)}{4} \sum_{j=1}^{4} E_{n-1}\left(P_{j}, R\right)
$$

or, upon passage to the limit,

$$
E(P, R)=\frac{k(P)}{4} \sum_{j=1}^{4} E\left(P_{j}, R\right) .
$$

This is readily seen to be equivalent with

$$
\Delta E+\frac{4(k-1)}{h^{2} k} E=0 \text { in } B .
$$

For a boundary point $S$ it follows from the definition of $e_{n}(P, R)$ that

$$
e_{0}(S, R)=\delta(S, R)
$$

if $\delta(S, R)$ denotes the function which is 0 for $S \neq R$ and 1 for $S=R$. For $n>0$ we have $e_{n}(S, R)=0$. These facts and eq 12 lead immediately to the statement of the theorem.

Remark 1. The function $E(P, R)$ can be considered the finite analog of the normal derivative of Green's function, since the solution that assumes the boundary values $f(R)$ can be written in the form $\sum_{R \in C} E(P, R) f(R)$ which recalls the formula

$$
u=\int_{C} \frac{\partial G(P, R)}{\partial n} f(R) d s
$$

from the theory of partial differential equations.

Remark 2. It is well known that the probability of a particle leaving the domain $B$ at a preassigned point $R$ of $C$ satisfies the difference eq 12 , if the particle is subject at each step to a probability of "dying" equal to $1-k(P)$. The corresponding function $g(P)$ is then everywhere nonpositive. In the next section it will be shown that the procedure of the present paper is applicable under considerably milder restrictions on $g(P)$. A further advantage of our method is that at each step only one random decision has to be made and not two as in a random walk involving the possibility of dying.

\section{Validity of the Procedure}

The statement of theorem 1 includes the assumption that $E(P, R)$ is finite. In this section we shall prove that this is the case, if and only if the eigenvalues of $\Delta u+g(P) u$ in $B$ are all positive. Here and in the sequel we mean by the eigenvalues of a linear difference operator $L[u]$ in the domain $B$ those values of $\lambda$ for which the problem

$$
L[u]+\lambda u=0 \text { in } B, u=0 \text { on } C
$$

has a nontrivial solution. The eigenvalues in this sense are the negative of the ergenvalues of the matrix formed by the left members of the system of linear equations represented by $L[u]=0$ in $B, u=0$ on $C$. It will simplify our terminology if we call the negative of this matrix the matrix "belonging to $L[u]$. ."

The proof of the theorem mentioned requires some preparations.

Lemma 1: The eigenvalues of $-\Delta u+\left(g-\frac{8}{h^{2}}\right) u$ are the same as those of $\Delta u+g u$.

Proof: The matrix $A$ belonging to

$$
\Delta u+g u=0 \text { in } B,
$$

is of the form $A=G+H$. Here, $G$ is a diagonal matrix formed with the values of $-g(P)+\frac{4}{h^{2}},(P$ in $B)$. 
The matrix $H$ has in the row belonging to any given point $P$, the element $-\frac{1}{h^{2}}$ in every column corresponding to an interior neighboring point of $P$, and zeros everywhere else. The matrix $A^{*}$ of the system $-\Delta u+\left(g-\frac{8}{h^{2}}\right) u=0$ is, similarly, given by $A^{*}=$

$G-H$. We may interpret these symmetric matrices as belonging to quadratic forms in the variables $u(P)$, $(P$ in $B)$. In the space of these variables consider the orthogonal transformation which consists in replacing the value of $u(P)$ by $-u(P)$ in every second point $P$ and leaving $u(P)$ unchanged in all the other points, in such a way that a point and its neighbors are always treated differently. This can, e. g., be done by setting

$$
u^{\prime}(P)=(-1)^{\frac{1}{h}(x+y)} u(P)
$$

where $(x, y)$ are the coordinates of $P$. This orthogonal transformation changes the quadratic form with matrix $G+H$ into the one with matrix $G-H$. These two matrices have, therefore, the same eigenvalues. by

Now we define an analytic function $\phi(P, R ; r)$ of $r$

$$
\phi(P, R ; r)=\sum_{n=0}^{\infty} r^{n} e_{n}(P, R) .
$$

We assume throughout that $g(P)<\frac{4}{h^{2}}$, and therefore $k(P)>0$. To show that the power series in definition 15 has positive radius of convergence consider the random walk problem in which $g(P)$ has been replaced everywhere by $\max _{P \in B} g(P)$, and denote by $e_{n}^{*}(P, R)$ and $k^{*}(P)$ the quantities corresponding to $e_{n}(P, R)$ and $k(P)$ in this new problem. Then, by the definition of $e_{n}(P, R)$,

$$
0 \leq e_{n}(P, R) \leq k^{* n}(P) p_{n}(P, R) \leq k^{*^{n}}
$$

where $p_{n}(P, R)$ is the probability of moving along a path that leads in exactly $n$ steps from $P$ to $R$. Hence, the radius of convergence of the series in formula 15 is at least equal to $1 / k^{*}$.

The formula 15 may, of course, be interpreted as the finite analogue of a Laplace transform, applied to the solution $e_{n}(P, R)$ of the "parabolic" difference equation 9. Pursuing this analogy, we find, by combining formulas 9 and 15 , for $\phi$ the elliptic difference equation

$$
k(\Delta \phi+g \phi)+\frac{4}{h^{2}}\left(1-\frac{1}{r}\right) \phi=0 .
$$

Our function $\phi$ is that solution of the difference eq 16 in $B$, which assumes the values $\delta(P, R)$ on the boundary $C$.

If we denote by $\Gamma$ the diagonal matrix whose elements are the values of $k(P)$ in $B$ and by $A$ the matrix belonging to $\Delta \phi+g \phi$, and if we set, for abbre- viation, $\frac{4}{h^{2}}\left(1-\frac{1}{r}\right)=\lambda$, then $\Gamma A-\lambda I$ is the matrix belonging to the difference eq 16 . The solutions of eq 16 are rational functions of $\lambda$ and therefore of $r$. It follows from definition 15 that $r=0$ is not a pole of this rational function.

For later use we mention that the poles of $\phi$ are simple. For if $D$ is a matrix such that $D^{2}=\Gamma$, we have $\Gamma A-\lambda I=D(D A D-\lambda I) D^{-1}$. The elements of the resolvent $(D A D-\lambda I)^{-1}$ of the symmetric matrix $D A D$ have only simple poles (see, e. g., [7], p. 26), and this property is not destroyed by the matrix transformation with the nonsingular matrix $D$. Hence, the elements of $(\Gamma A-\lambda I)^{-1}$ have only simple poles as functions of $\lambda$, i. e., $\phi$ has only simple poles as functions of $r$.

Lemma 2. The number $\frac{4}{h^{2}}\left(1-\frac{1}{r_{1}}\right)$ is an eigenvalue of $k(\Delta \phi+g \phi)$, if and only if $r=r_{1}$ is a pole of $\phi(P, R ; r)$ for at least one pair of points $P, R$.

Proof. If $r=r_{1}$ is a pole of $\phi(P, R ; r)$ for some $P$ and $R$ then $\frac{4}{h^{2}}\left(1-\frac{1}{r_{1}}\right)$ is a pole of some element of the resolvent of the matrix belonging to $k(\Delta \phi+g \phi)$. Hence, this number is an eigenvalue of that matrix.

Conversely, let $\frac{4}{h^{2}}\left(1-\frac{1}{r_{1}}\right)$ be an eigenvalue of $k(\Delta \phi+g \phi)$ and assume that $\phi\left(P, R ; r_{1}\right)$ exists for all $P$ and $R$. In order to show that this implies a contradiction we make use of Green's formulas for the difference operator $\Delta u$. These formulas are (cf. [1])

$$
h \sum_{B+C}\left[u_{x} v_{x}+u_{y} v_{y}\right]=-h \sum_{B} v \Delta u-\sum_{C} v \Gamma(u)
$$

and

$$
h \sum_{B+C}[v \Delta u-u \Delta v]+\sum_{C}[v \Gamma(u)-u \Gamma(v)]=0
$$

where $u_{x}, u_{y}$, etc., denote the first forward differences and where the expression $\Gamma(u)$ is defined as follows: if $u$ assumes the value $u_{0}$ at a given boundary point $R$ and the values $u_{1}, \ldots, u_{\nu}(\nu \leqslant 3)$ at the $\gamma$ neighboring points of $R$ in $B+C$, then

$$
\Gamma(u)=\frac{1}{h}\left(u_{1}+\ldots+u_{\nu}-\nu u_{0}\right) .
$$

Green's formulas 17 and 18 are valid for any functions that vanish outside of $B+C$. If we substitute in the identity 18 for $v$ the function $\phi\left(P, R ; r_{1}\right)$ and for $u$ an eigenvector $U$ corresponding to the eigenvalue $\frac{4}{h^{2}}\left(1-\frac{1}{r_{1}}\right)$, it follows that $\Gamma(U)=0$, identically for all boundary points $R$. Substituting $U$ for $u$ in the identity 17 we see that the bilinear form

$$
\sum_{B+C}\left\{U_{x} v_{x}+U_{y} v_{y}-\left[g+\frac{4}{h^{2} k}\left(1-\frac{1}{r_{1}}\right)\right] U v\right\}=0
$$

vanishes identically for any choice of the function $v$. Taking for $v$ the function that is 1 at an arbitrary 
interior point and 0 everywhere else, the identity 20 is seen to imply for $U$ the first order difference equation

$U_{x}+U_{y}+h\left[g+\frac{4}{h^{2} k}\left(1-\frac{1}{r_{1}}\right)\right] U=0$ in $B, U=0$ on $C$.

Together with

$$
k(\Delta U+g U)+\frac{4}{h^{2}}\left(1-\frac{1}{r_{1}}\right) U=0
$$

this yields

$$
h \Delta U-U_{x}-U_{y}=0 \text { in } B, U=0 \text { on } C,
$$

i. e.

$U(x-h, y)+U(x, y-h)-2 U(x, y)=0$ in $B, U=0$ on $C$.

This last difference equation has the unique solution $U=0$ as can be seen, e. g., by calculating its values from point to point from the boundary inwards.

Since $U$ is, by assumption, not identically zero we have arrived at a contradiction, and the proof of the lemma is completed. We are now ready to formulate and prove our theorem.

Theorem 2: If $g(P)<\frac{4}{h^{2}}$ in $B$, then $E(P, R)=$ $\lim E_{n}(P, R)$ is finite for all $P$ in $B$ and all $R$ on $C$, if $\stackrel{n \rightarrow \infty}{\text { and only if all eigenvalues of } \Delta u+g u \text { are positive. }}$

Proof: The difference expression $k(\Delta u+g u)$ is, in general, not self-adjoint, i. e. the matrix belonging to it is, in general, not symmetric. But its eigenvalues, i. e. the numbers $\lambda$ for which the problem

$$
\Delta u+g u+\lambda k^{-1}(P) u=0 \text { in } B, u=0 \text { on } C
$$

has a nontrivial solution are positive if and only if those of $\Delta u+g u$ are. In fact, let $A$ be the metrix belonging to $\Delta u+g u$ and denote by $D$ the diagonal matrix whose elements are the positive determinations of $k^{\frac{1}{2}}(P)$. Then eq 22 can be written in the form

$$
A u=\lambda D^{-2} u \text {. }
$$

If we make the substitution

$$
u=D v
$$

this is equivalent with

$$
D A D v=\lambda v .
$$

Hence $\lambda$ is an eigenvalue of the symmetric matrix $D A D$ and therefore real. If we write $D A D^{\prime}$ instead of $D A D$ we see that $D A D^{\prime}$ is the matrix of the quadratic form obtained from the one whose matrix is $A$ by the transformation 23 . Since this transformation does not destroy the positive definite character of a quadratic form, and the same is true of its inverse, $A$ is positive definite, if and only if all the eigenvalues of eq 22 are positive.
By lemma 2, the eigenvalues of $k(\Delta u+g u)$ are all positive if and only if $\frac{4}{h^{2}}\left(1-\frac{1}{r}\right)$ is positive for all poles of $\phi(P, R ; r)$, i. e. if these poles are all greater than $r=1$, or negative. On the other hand, by the definition of $\phi(P, R ; r)$ the expected mass $E(P, R ; r)$ is finite, if and only if all poles of $\phi$ are numerically greater than 1. Our proof will be completed, if we show that the occurrence of negative poles greater than or equal to -1 implies the existence of nonpositive eigenvalues of $\Delta u+g u$. To show this we substitute $\lambda=\lambda *+\frac{8}{h^{2}}$ into eq 22 and obtain, after a short calculation using the expression 3 for $k(P)$,

$$
k\left[\Delta u+\left(\frac{8}{h^{2}}-g\right) u\right]+\lambda * u=0 \text { in } B, u=0 \text { on } C .
$$

In view of lemma 1 and the first part of this proof, the existence of nonnegative eigenvalues $\lambda^{*}$ is equivalent with the existence of nonpositive eigenvalues of $\Delta u+g u$. On the other hand, $\lambda^{*}=0$ means $\lambda \geq \frac{8}{h^{2}}$ and by lemma 2 such a $\lambda$ is an eigenvalue of $k(\Delta u+g u)$, if and only if $\phi(P, R ; r)$ has a pole in $-1 \leqslant r<0$. This completes the proof.

Corollary: If

$$
g(P)=\rho-V(P)
$$

where $\rho$ is a parameter, $\lim _{n \rightarrow \infty} E_{n}(P, R)$ exists, if and only if $\rho$ is less than the lowest eigenvalue of $\Delta u$ $V(P) u$.

Proof: The eigenvalues of $\Delta u-V(P) u$ are obtained from those of $\Delta u+(\rho-V(P) u$ by subtracting $\rho$. Therefore the eigenvalues of the latter expression are positive, if and only if those of $\Delta u-V u$ exceed $\rho$.

\section{Practical Bound for Validity of Random Walk Procedure}

If the method described in the preceding section is to be used, we have to be sure that the condition of theorem 2 is satisfied. Since the eigenvalues are, in general, not known, the following sufficient criterion may be useful.

Theorem 3: The expected value $E(P, R)$ exists, if in $B$

$$
g(P) \leq \pi^{2}\left(\frac{1}{a^{2}}+\frac{1}{b^{2}}\right)-\frac{\pi^{4}}{12} h^{2}\left(\frac{1}{a^{4}}+\frac{1}{b^{4}}\right)
$$

where $a$ and $b$ are the sides of the smallest rectangle with sides parallel to the axes that contains $B$.

Proof: We substitute for the problem defined by formulas 1 and 2 the similar problem obtained by replacing $g(P)$ everywhere by its maximum $\gamma$ in $B$, by replacing the domain $B$ by the circumscribed rectangle $B_{1}$ with sides $a$ and $b$, and finally by imposing the boundary condition: $u==1$ on the boundary $C_{1}$ of $B_{1}$. Consider the mass upon arrival anywhere on $C_{1}$ for this new problem. Its expected value, 
$E_{1}(P)$ - if it is finite - is at least equal to the expected value $E(P, R)$ of the original problem, and it solves the modified difference equation problem.

From theorem 2 we know that $E_{1}(P)$ is finite, if $\Delta u+\gamma u-0$ has only positive eigenvalues corresponding to the domain $B_{1}$, i. e. if the smallest eigenvalue $\rho_{1}$ of $\Delta u=0$ exceeds $\gamma$.

The eigenvalues $\rho_{j}$ of $\Delta u=0$ in a rectangle can be calculated in literal analogy to the familiar procedure for the Laplace equation. (See [2], p. 258). They turn out to be the values of

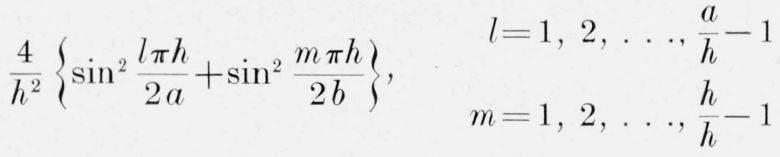

Setting $l=m=1$ and increasing the expression by substituting the first two terms of the power series for the sines the proof of our theorem is at hand.

\section{Green's Function}

Using the procedure and the notation of the previous sections let $g_{n}(P, Q)$ be the expected amount of mass that passes through an inner point $Q$ on the $n$-th step of a walk that starts at $P$ and ends on the boundary $C$. Clearly,

$g_{0}(P, Q)=\delta(P, Q), g_{n}(R, Q)=0,(n>0), R$ on $C$.

The difference equation

$$
g_{n}(P, Q)=\frac{k(P)}{4} \sum_{j=1}^{4} g_{n-1}\left(P_{j}, Q\right), n>0
$$

for $g_{n}(P, Q)$ is derived precisely like its analog, eq 9 . If we set

$$
G_{n}(P, Q)=\sum_{\nu=0}^{n} g_{\nu}(P, Q)
$$

and

$$
G(P, Q)=\lim _{n \rightarrow \infty} G_{n}(P, Q),
$$

then $G(P, Q)$, if it is finite, solves the problem

$$
\Delta u+g(P) u=-\frac{4}{h^{2} k(P)} \delta(P, Q) \text { in } B, u=0 \text { on } C
$$

which corresponds to eq 12 . When there is no indication to the contrary the symbol $\Delta$ is always meant to operate on the point $P$.

The solution of

$$
\Delta u+g u+f(P)=0 \text { in } B, u=0 \text { on } C
$$

can be written in the form

$$
u(P)=\frac{h^{2}}{4} \sum_{r_{i \in B}} G(P, Q) k(Q) f(Q) .
$$

In analogy with the terminology for differential equations we shall call the function

$$
K(P, Q)=\frac{h^{2}}{4} k(Q) G(P, Q)
$$

Green's function for our difference equation and the domain $B$.

The following experiment leads to a random variable whose expected value is $u(P)$ provided $G(P, Q)$ is finite.

On every step of the random walk multiply the amount of mass at the instant by the value of $k(Q) f(Q)$ at that particular point and add the products thus obtained. If the cumulative sum after $N$ random walks starting at $P$ is multiplied by $\frac{h^{2}}{4 N}$, we have an estimate of $u(P)$.

As to the existence of $G(P, Q)$ we have a theorem analogous to theorem 2 .

Theorem 4: If $g(P)<\frac{4}{h^{2}}$ in $B$, then $\lim _{n \rightarrow \infty} G_{n}(P, Q)$ is finite for all $P$ and $Q$ in $B$, if and only if all eigenvalues of $\Delta u+g u$ in $B$ are positive.

Proof: Define $\psi(P, Q ; r)$ by

$$
\psi(P, Q ; r)=\sum_{n=0}^{\infty} r^{n} g_{n}(P, Q) .
$$

The convergence for sufficiently small $|r|$ is shown exactly as in the case of the function $\phi(P, R ; r)$. From formulas 25 and $26, \psi$ is seen to solve the problem

$k(\Lambda \psi+g \psi)+\frac{4}{h^{2}}\left(1-\frac{1}{r}\right) \psi=-\frac{4}{h^{2} r} \delta(P, Q)$ in $B, \psi=0$ on $C$.

It is therefore a rational function of $r$. The poles of $r$ and the eigenvalues $\frac{4}{h^{2}}\left(1-\frac{1}{r}\right)$ of $k(\Delta \psi+g \psi)$ are shown to correspond to each ocher, just as in the proof of lemma 2. The reasoning is somewhat simpler here, since instead of $\Gamma(U)=0$ we obtain here immediately $\sum_{P \in B} U(P) \delta(P, Q)=0$. If this is true for all $Q$ in $B$, it follows that $U(P)=0$ and the contradiction is at hand. The subsequent reasoning is precisely the same as for theorem 2.

\section{Random Walks in Unbounded Domains}

If $B$ is an unbounded domain other than the full plane, then the quantity $E(P, R)$ may still exist. The function $G(P, Q)$, on the other hand, and its probabilistic interpretation may exist even in the full plane. We limit ourselves, therefore, to a discussion of this latter quantity.

Some facts from the theory of infinite matrices will have to be used. For convenience we summarize all the definitions and properties used, and add references to the corresponding pages of [7]. 
(a) If $A=\left\{a_{i k}\right\},(i, k=1,2, \cdots)$, is an infinite matrix, the finite matrices $A_{j}$ obtained from $A$ by letting $i$ and $k$ run from 1 to $j$ only are called the segment matrices of $A$, (p. 121). The set consisting of all eigenvalues of all segment matrices and their accumulation points is called the segment spectrum of $A$, (p. 124).

(b) An infinite vector $x_{i},(i=1,2, \cdots)$ is called quadratically convergent, if $\sum_{i=1}^{\infty}\left|x_{i}\right|^{2}$ is finite, (p. 125).

(c) An infinite matrix is called bounded, if there is a constant $M$ independent of $n$ such that $\left(\sum_{i, k=1}^{n} a_{i k} x_{i} y_{k}\right)^{2} \leqq M \cdot \sum_{i=1}^{\infty}\left|x_{i}\right|^{2} \cdot \sum_{i=1}^{\infty}\left|y_{i}\right|^{2},($ p. 124). The product of two bounded matrices exists and is bounded, (p. 131). For the multiplication of bounded matrices the associative law is true, (p. 131). If the segment matrices are normal, i. e., if $A_{j} \bar{A}_{j}^{\prime}=$ $A_{i}^{\prime} A_{j}$, then $A$ is bounded, if and only if its segment spectrum is bounded, (p. 124).

(d) An infinite Hermitian matrix is called positive definite, if there is a positive constant $\mu$, independent of $n$, such that for all $x_{i}$

$$
\sum_{i, k=1}^{n} a_{i k} x_{i} \bar{x}_{k} \leq \mu \sum_{i=1}^{n}\left|x_{i}\right|^{2}, \text { (p. 124). }
$$

It is called nonnegative definite, if

$$
\sum_{i, k=1}^{n} a_{i k} x_{i} \bar{x}_{k} \geq 0 \text {, for all } n \text { and all } x_{i}, \text { (p. 124). }
$$

(e) If there exists a constant $C$ such that $\sum_{k=1}^{\infty}\left|a_{i k}\right|$ $<C, \sum_{k=1}^{\infty}\left|a_{k i}\right|<C, \quad(i=1,2, \cdots)$, then the infinite matrix $A=\left(a_{i k}\right)$ is bounded (p. 153).

(f) If $A$ is bounded and normal, then it possesses a bounded inverse if and only if $A \bar{A}^{\prime}$ is positive definite (p. 138).

(g) If $A=\left(a_{\imath k}\right)$ is positive definite, then it possesses a bounded inverse $A^{-1}=\left(\alpha_{i k}\right)$ and, if $A_{j}^{-1}=\left(\alpha_{i k}^{(j)}\right)$ is the inverse of the segment matrix $\mathbf{A}_{j}$, then

$$
\alpha_{i k}=\lim _{j \rightarrow \infty} \alpha_{i k}^{(i)} \text { for all } i \text { and } k, \text { (p. 229). }
$$

(h) If $A^{2}$ is positive definite and $A$ is nonnegative definite, then $A$ is positive definite.

The definitions at the beginning of section 4 can be applied without significant change to an unbounded domain.

Let the points of the domain be numbered in such a way that the first $j$ points form, for every $j$, a simply connected domain $B_{j}$ of lattice points. If the expected value $G(P, \cap)$ is finite for a given pair of points $P$ and $R$, then the expected value $G^{(j)}(P, P)$ exists for the corresponding random walk problem in all those domains $B_{j}$ which contain $P$ and $r_{j}$, i. e., for all sufficiently large $j$. One has, furthermore,

$$
\lim _{j \rightarrow \infty} G^{(j)}(P, Q)=G(P, Q) .
$$

We shall call the expression $\Delta u+g u$ positive definite, if the real and symmetric infinite matrix $A$ belonging to it is positive definite. In this matrix the rows and columns will always be assumed to be arranged in the order of the numbering of the points of $B$ which was described above. (But the positive definite character of an infinite matrix is, of course, not destroyed if the rows and columns are subjected to the same rearrangement.)

Theorem 5: If $\Delta u+g u$ is positive definite in the unbounded domain $B$, and if $g(P) \leq \frac{4}{h^{2}}$ in $B$, then the expected value $G(P, Q)$ exists.

Proof: Since $\Delta u+g u$ is positive definite in $B$, it is a fortiori positive definite in all domains $B_{j}$. The matrix belonging to $\Delta u+g u$ in $B_{j}$ is the segment matrix $A_{j}$ of $A$. By theorem 4, the expected value $\mathrm{G}^{(j)}(P, Q)$ is finite,-provided $P, Q$ are points of $B_{j}$ - and solves problem eq 29. Denote by $A_{j}(P, Q)$ the elements of the matrix $A_{j}$ in the row and column corresponding to $P$ and $Q$, respectively, and by $A_{i}^{(-1)}(P, Q)$ the corresponding element of the inverse matrix. Then

$$
\begin{aligned}
G^{(j)}(P, Q) & =\frac{4}{h^{2}} \sum_{S \in B_{j}} A_{j}^{(-1)}(P, S) \cdot k^{-1}(S) \delta(S, Q) \\
& =\frac{4}{h^{2}} A_{j}^{(-1)}(P, Q) k^{-1}(Q)
\end{aligned}
$$

From this equality we conclude, by virtue of relation 35 , and of the property $(g)$ stated above, that $C(P, Q)$ exists and is given by

$$
G(P, Q)=\frac{4}{h^{2}} A^{(-1)}(P, Q) k^{-1}(Q)
$$

where $A^{(-1)}(P, Q)$ are the elements of the matrix $A^{-1}$.

Corollary: If, in addition to the assumption of theorem 5 , it is known that $g(P) \geq$ const. $>-\infty$ in $B$, then $G(P, Q)$, as an infinite matrix, is bounded.

Proof: For then, $0<k^{-1}(Q) \leq$ const. $<\infty$ in $B$, and the right member of eq $3 \overline{6}$ defines a bounded matrix, since $A^{-1}$ is bounded by virtue of $(g)$.

Theorem 6: If $G(P, Q)$ exists for all $P$ and $Q$, then $\Delta u+g u$ is nonnegative definite.

Proof: If $G(P, Q)$ is finite, so are all $G^{(j)}(P, Q)$, and therefore all segment matrices $A_{j}$ are positive definite by theorem 2, hence $A$ is nonnegative definite, by definition $(d)$.

Theorem 6 cannot be strengthened to a full converse of theorem 5. This can easily be seen from the following example. Let $g(P)$ be zero, so that $k(P)=1$ and eq 1 reduces to $\Delta u=0$. Then $G(P, Q)$ is simply the expected number of visits at ? for a moving point starting from $P$ and being absorbed as soon as it meets the boundary $C$ for the first time. This quantity is known to be finite for an infinite quadrant but infinite for the whole plane (see [5] ), although $\Delta u$ is semidefinite in both these domains, in consequence of the results of section 4 . But the following weaker statement can be proved. 
Theorem 7: Assume that $G(P, Q)$ exists and is a bounded infinite matrix. If then $g(P)$ satisfies in the unbounded domain $B$ the inequality

$$
g(P) \leqslant \frac{4}{h^{2}}-\epsilon,(\epsilon>0)
$$

then $\Delta u+g u$ is positive definite in $B$.

Proof: If $G(P, Q)$ exists then it satisfies the difference equation 29 in $B$, i. e., if $A(P, Q)$ are the elements of $A$,

$$
\sum_{S \in B} A(P, S) G(S, Q)=\frac{4}{h^{2}} \delta(P, Q) k^{-1}(P) .
$$

In other words, if $\Gamma$ denotes the infinite diagonal matrix formed with the elements $k(P)$ and $G$ denotes the infinite matrix with elements $G(P, Q)$, then $\frac{h^{2}}{4} G \Gamma$ is a right inverse of $A$. The matrix $A$ is bounded, thanks to assumption 37 and property $(e)$. Since $\Gamma$ is bounded, because of assumption 37, the matrix $\frac{h^{2}}{4} G \Gamma$ is bounded in consequence of property (e). Thus, $A$ is bounded and normal (even symmetric) and possesses a bounded inverse. Therefore, $A^{2}$ is positive definite by property $(f)$, and property $(h)$ assures the positive definiteness of $A$ itself.

Remarks: In view of the facts mentioned after the proof of theorem 6 , it follows from theorem 7 that the expected number of visits at a point $Q$ during a random walk starting from a point $P$ of an infinite quadrant forms an infinite matrix that is unbounded.

\section{Sampling Method for Calculation of Lowest Eigenvalue}

The fact that the radius of convergence of the functions $\phi$ of formula 15 and $\psi$ of formula 33 determine the lowest eigenvalue of $k(\Delta u+g u)$ can be used for an approximate experimental determination of this eigenvalue. This gives us an approximation to the lowest eigenvalue of the differential expression $\frac{\partial^{2} u}{\partial x^{2}}+\frac{\partial^{2} u}{\partial y^{2}}+g u$ in the domain $B$ or in any domain $B^{\prime}$ bounded by a piecewise smooth curve $C^{\prime}$ and containing in its interior the same lattice points as $B$. For $k$ approaches 1 as fast as $h^{2}$, as $h \rightarrow 0$, and the eigenvalues of $\Delta u+g u$ approach those of the corresponding differential equation, (cf. [1]).

The following lemma is needed for the subsequent discussion:

Lemma 3: The poles of the function $\phi(P, R ; r)$ (and also those of $\psi(P, Q ; r))$ lie symmetrically with respect to $r=0$. This is equivalent to the statement that the eigenvalues of $k(\Delta u+g u)$ lie symmetrically with respect to $\lambda=\frac{4}{h^{2}}$.

Proof: The argument applied in the proof of lemma 1 to the matrix $A$ belonging to $\Delta u+g u$ can be literally extended to the matrix $D A D$ of the proof of theorem 2 , with the result that the eigenvalues of $k(\Delta u+g u)$ are the same as those of $-k\left[\Delta u+\left(g-\frac{8}{h^{2}}\right) u\right]$. But in the proof of theorem 2 it was also shown that, if $\lambda$ is an eigenvalue of $k(\Delta u+g u)$, then $\frac{8}{h^{2}}-\lambda$ is an eigenvalue of $-k\left[\Delta u+\left(g-\frac{8}{h^{2}}\right) u\right]$. Combining these two facts the proof of the second statement of our lemma is at hand. The first statement is equivalent to the second by virtue of lemma 2 .

We first discuss the use of the function $\phi$ for our purpose. The numerical computations will be simpler if, instead of operating with $\phi$ directly, the function

$$
\phi^{*}(P, r)=\sum_{R \in C} \phi(P, R ; r)
$$

is used. If we define $e_{n}^{*}(P)$ by

$$
e_{n}^{*}(P)=\sum_{R \in C} e_{n}(P, R),
$$

then formula 15 implies

$$
\phi^{*}(P)=\sum_{n=0}^{\infty} r^{n} e_{n}^{*}(P) .
$$

The function $\phi^{*}(P)$ satisfies the difference equation 16 in $B$ and assumes the boundary value

$$
\phi^{*}(R)=1, \text { for } R \text { on } C .
$$

Let $\lambda_{1}$ be the lowest eigenvalue of $k(\Delta u+g u)$. By lemma 2 and lemma 3 the radius of convergence of $\phi(P, R ; r)$ is then

$$
r_{1}=\left(1-\frac{h^{2}}{4} \lambda_{1}\right)^{-1}
$$

The radius of convergence of $\phi^{*}(P, r)$ is, in general, also $r_{1}$. Exceptionally, it may be larger, owing to cancellation of poles in the sum $\sum_{R \in C} \phi(P, R ; r)$. In this case the method to be described would lead to some higher, rather than to the lowest eigenvalue. If this is suspected, the result may be checked by repeating the computations with a different $P$ or with formula 15 directly. We shall exclude this case from our considerations.

The quantity $e_{n}^{*}(P)$ is the expected mass upon arrival anywhere on the boundary for random walks starting at $P$ and consisting of exactly $n$ steps. By performing a sufficient number of random walks starting at $\mathrm{P}$ those $e_{n}^{*}(P)$ for which $n$ is not too large can be estimated experimentally.

In order to find $r_{1}$ from these data we recall that $\phi^{*}(P)$ is a rational function whose poles are real and symmetric with respect to the origin. Hence, it is of the form

$$
\phi^{*}(P ; r)=\sum_{\nu \leq N / 2}\left[\frac{c_{\nu}(P)}{r-r_{\nu}}+\frac{c_{-\nu}(P)}{r+r_{\nu}}\right]+r d_{1}(P)+d_{2}(P) .
$$


Here, $N$ is the number of points in B. For even $N$ the quantity $d_{1}(P)$ is zero. $c_{1}(P)$ is, by assumption, not zero for the point $P$ chosen. From formulas 38 and 39 we find

$$
e_{n}^{*}(P)=\sum_{\nu \leq N / 2} r_{\nu}^{-n}\left[c_{\nu}(P)+(-1)^{n} e_{-\nu}(P)\right], \quad(n>2) .
$$

If $n$ is not too small, we can write, with sufficient approximation,

$$
e_{n}^{*}(P) \sim r_{1}^{-n}\left[c_{1}(P)+(-1)^{n} c_{-1}(P)\right],
$$

i. e.,

$$
r_{1}^{2} \sim e_{n}^{*}(P) / e_{n+2}^{*}(P) .
$$

By using several values of $n$, the estimate of $r$ can be improved, and at the same time the admissibility of the approximation used is tested. The lowest eigenvalue is then given by

$$
\lambda_{1}=\frac{4}{h^{2}}\left(1-\frac{1}{r_{1}}\right)
$$

The function $\psi$ of section 5 can also be used to compute $\lambda_{1}$. One defines $\psi^{*}(P, r)$ and $g_{n}^{*}(P, r)$ by

$$
\psi^{*}(P, r)=\sum_{Q \in B} \psi(P, Q ; r),
$$

and

$$
g_{n}^{*}(P)=\sum_{Q \in B} g_{n}(P, Q ; r)
$$

Then $\psi^{*}(P, r)=\sum_{n=0}^{\infty} r^{n} g_{n}^{*}(P) . \quad g_{n}^{*}(P)$ is the expected mass anywhere in $B$ at the $n$th step of a random walk starting at $P$. This quantity can be found experimentally by performing a sufficient number of random walks starting from $P$, and recording the mass at every step. The sum of all recorded masses for the $n$th step divided by the total number of walks performed is an approximate value for $g_{n}^{*}(P)$. From thereon the procedure is exactly like the one described for $\phi^{*}(P ; r)$.

If the domain $B$ is unbounded, the concept of the lowest eigenvalue has to be replaced by that of the left endpoint of the speetrum, which may, of course, be at $-\infty$. We limit the discussion to cases in which the spectrum is bounded from below.

Since no experimental method can take into account random walks beyond a certain length, all experiments take place in some finite subdomain $B_{j}$ of $B$, so that we must answer the question whether the results thus obtained approximate the correct values for the given unbounded domain. This question is answered affirmatively by the theorem that the left endpoint of the segment spectrum coincides with the left endpoint of the spectrum, provided the matrix is halfbounded and symmetric ([7] p. 231).

\section{Comparison with Method of Donsker and $\mathrm{Kac}$}

In this section we shall assume that we are dealing with a one dimensional problem. This will enable us to add a few heuristic remarks on the relationship between our method and that of Donsker and Kac, [3], without introducing notational complications.

Let $x_{j}$ be the position of a point at the $j$ th step of a random walk in a linear net of mesh length $h$. The particle is supposed to start from $x=0$ and to be equally likely to choose either of its two neighbors at each step. Then $x_{j}$ is the sum of $j$ identically and independently distributed random variables with mean 0 and standard deviation $h$. Donsker and Kac are primarily concerned with the limiting distribution, as $h \rightarrow 0$, of a random variable which, in our notation, can be written (our $g(x)$ is $-2 V(x)$ in their notation)

$$
\alpha(h, t)=-\frac{h^{2}}{2} \sum_{j \leq t / h^{2}} g\left(x_{j}\right) .
$$

Here $t$ is an arbitrary positive parameter.

The quantity $g_{n}^{*}(P)$ of section 7 is, in one dimension and for $P=0$, the expected value of the random variable

$$
\gamma(h, n)=\prod_{j \leq n} k\left(x_{j}\right)=\prod_{j \leq n}\left(1-\frac{h^{2}}{2} g(x)_{j}\right)^{-1}
$$

For small $h$, and with

$$
t=h^{2} n
$$

we have, approximately,

$$
\gamma(h, n) \sim e^{-\alpha(h, t)} .
$$

Our function $\psi^{*}(P, r)$ of section 7 is the expected value of the random variable

$$
\sum_{n=0}^{\infty} r^{n} \gamma(h, n)
$$

With the substitution

$$
r=e^{-s h^{2}}
$$

and using relation 40 it is seen that, approximately for small $h$,

$$
\sum_{n=0}^{\infty} r^{n} \gamma(h, n) \sim \frac{1}{h^{2}} \int_{0}^{\infty} e^{-s t-\alpha(h, t)} d t .
$$

If $\sigma(h, \alpha, t)$ is the distribution function of $\alpha(h, t)$, we have, therefore,

$$
\psi^{*}(P, r) \sim \frac{1}{h^{2}} \int_{0}^{\infty} \int_{0}^{\infty} e^{-\alpha(h, t)-s t} d_{\alpha} \sigma(h, t) .
$$

On the other hand, $\psi^{*}(P, r)$ satisfies a difference equation problem which differs from eq 34 only in 
that the factor $\delta(P, D)$ in the right member is to be replaced by 1 and the factors 4 by 2 . This last change is necessary because we are dealing now with the onedimensional problem. Because of eq 42 we may write for small $h$,

$$
1-\frac{1}{r} \sim-s h^{2}, r \sim 1 .
$$

Furthermore, $k \sim 1$, and the difference equation may be replaced by the corresponding differential equation. With these changes, we obtain from the difference equation 34 the differential problem

$$
\frac{d^{2} u}{d x^{2}}+(g-2 s) u+\frac{2}{h^{2}}=0 \text { in } B, u=0 \text { on } C,
$$

whose solution will be approximately $\psi^{*}$. In the paper by Kac $B$ is the whole line. In this case, the solution of eq $34^{*}$ at $x=0$ can be written in the form

$$
u=\frac{1}{h^{2}} \int_{-\infty}^{\infty} \chi(x) d x
$$

where $\chi(x)$ is Green's function on the whole line for the differential equation

$$
\frac{1}{2} \frac{d^{2} u}{d x^{2}}+\left(\frac{g}{2}-s\right) u=0
$$

For small $h$, the left members of eq 43 and 44 are approximately equal. If we denote by $\sigma(\alpha, t)$ the limit of the distribution function $\sigma(h, \alpha, t)$, as $h \rightarrow 0$, it seems therefore plausible that the identity

$$
\int_{0}^{\infty} \int_{0}^{\infty} e^{-\alpha-s t} d \alpha \sigma(\alpha, t) d t=\int_{-\infty}^{\infty} \chi(x) d x
$$

will hold exactly. This identity, proved by Kac in [4], is the theoretical basis of the method of Donsker and Kac in [3].

In [4] the passage to the limit, as $h \rightarrow 0$, is essential, since that paper is concerned with the distribution of certain Wiener integrals. The sampling method of [3] operates, of course, with finite sums only. The methods of [3] and those of the present paper are therefore closely related. In order to be sure that the results obtained approximate those for the corresponding differential equation we refer to [1]. The analogous part of Kac's theory in [4] also makes use of the methods of [1].

Dated: March 21, 1950.

\section{References}

[1] R. Courant, K. Friedrichs, H. Lewy, Ueber die partiellen Differenzengleichungen der mathematischen Physik, Math. Ann. 100, 32 to 74 (1928).

[2] R. Courant and D. Hilbert, Methoden der mathematischen Physik, 1 (Berlin, 1931).

[3] M. D. Donsker and M. Kac, A sampling method for determining the lowest eigenvalue and the principal eigenfunction of Schroedinger's equation, to be published in J. Research NBS.

[4] M. Kac, On distributions of certain Wiener functionals, Trans. Amer. Math. Soc. 65, 1 to 13 (1949).

[5] W. H. McCrea and F. J. Whipple, Random paths in two and three dimensions, Proc. Roy. Soc. Edinburgh, 60, 281 to $298(1939-40)$.

[6] J. Petrovsky, Ueber das Irrfahrtproblem, Mathematische Ann. 109, 425 to 444 (1934).

[7] A. Wintner, Spektraltheorie der unendlichen Matrizen (Leipzig, 1929).

Los Angeles, March 31, 1950. 